$$
\text { punto org }
$$

Collana diretta da Luigi Maria Sicca

89 


\title{
LA PROGETTAZIONE DEI SISTEMI ORGANIZZATIVI PER LA TRASFORMAZIONE DIGITALE
}

\author{
Presentazione \\ Luigi Nicolais \\ Prefazione \\ Raffaella Cagliano \\ Postfazione \\ Luigi Maria Sicca
}

Editoriale Scientifica

Napoli 
Tutti i diritti sono riservati

(C) Copyright 2021 Editoriale Scientifica s.r.1.

Via San Biagio dei Librai, 39 - 80138 Napoli

www.editorialescientifica.com info@editorialescientifica.com

ISBN 979-12-5976-101-9 


\section{Indice}

9 Presentazione

Luigi Nicolais

13 Prefazione

Raffaella Cagliano

17 INTRODUZione

21 1. OrganizZaZIONE E PROgETtAZIONE ORganizZATIVA: CONCETTI DI BASE

$23 \quad 1.1$ Cosa intendiamo per organizzazione

$25 \quad 1.2$ Organizzazione, razionalità e progettazione

$27 \quad 1.3 \quad$ Il problema organizzativo

$31 \quad 1.4$ Scopo delle organizzazioni e obiettivi organizzativi

$33 \quad 1.5$ Teoria e progettazione organizzativa

35 2. LA PROGETTAZIONE NELL'EVOLUZIONE DEL PENSIERO ORGANIZZATIVO

$38 \quad 2.1 \quad$ La scuola classica

38 2.1.1 Dalle origini della teoria organizzativa alla scuola classica

39

2.1.2 Il contesto storico della scuola classica

41

2.1.3 Taylor e l'organizzazione scientifica del lavoro

43

2.1.4 L'organizzazione scientifica del lavoro dopo Taylor

45

2.1.5 Critiche allo scientific management

46

2.1.6 Fayol e i principi di direzione

50

2.1.7 Weber e la burocrazia 
51

51

53

56

56

65

73

73

76

76

77

77

80

82

84

95

95

99

101

103

105

107

113

113

114

115

116
2.2 Dal focus sul sistema tecnico al focus sul sistema sociale e sull'individuo

2.2.1 La scuola delle relazioni umane

2.2.2 Risorse umane e motivazione

2.3 La fase della maturità nelle teorie organizzative

2.3.1 L'organizzazione come sistema aperto

2.3.2 Decisioni, azioni e informazioni nella progettazione organizzativa

2.4 Prospettive teoriche sulle relazioni inter-organizzative 2.4.1 Gerarchia, mercato e reti

2.4.2 L'ecologia delle popolazioni organizzative

2.4.3 La dipendenza da risorse

2.5 Sviluppi ulteriori del pensiero organizzativo

2.5.1 L'organizzazione snella

2.5.2 L'apprendimento organizzativo

2.5.3 Resource based view e knowledge based view

2.6 Evoluzione della progettazione organizzativa

3. Il sistema organizzativo

3.1 L'organizzazione come sistema

3.2 Il sistema organizzativo e suoi livelli

3.2.1 La struttura organizzativa generale

3.2.2 I processi aziendali

3.2.3 La posizione di lavoro

3.3 L'interdipendenza tra i livelli del sistema organizzativo

3.4 Le performance del sistema organizzativo

3.4.1 La performance al livello di struttura organizzativa generale

3.4.2 La performance al livello di processo

3.4.3 La performance al livello di posizione di lavoro

3.4.4 L'integrazione tra i tre livelli di performance 
119 4. LA PROGETTAZIONE DEL SISTEMA ORGANIZZATIVO

1204.1 La progettazione della struttura organizzativa generale

120 4.1.1 I criteri per la scelta della tipologia della struttura organizzativa generale

4.1.2 La struttura funzionale

124

4.1.3 La struttura divisionale

4.1.4 La struttura per progetti

4.1.5 La struttura a matrice

4.1.6 La struttura per processi

4.1.7 Il confronto tra le diverse tipologie di struttura ed $i$ criteri per la loro scelta

4.1.8 L'evoluzione della struttura organizzativa nella vita dell'impresa

4.1.9 Identificazione delle aree di responsabilità

4.1.10 Identificazione e dimensionamento delle aree di responsabilità di tipo funzionale

4.1.11 Identificazione delle attività elementari

4.1.12 Valutazione delle relazioni tra attività elementari

4.1.13 La definizione dei nuclei omogenei di attività

4.1.14 La valutazione del carico di lavoro ed il dimensionamento delle unità organizzative

4.4 Alcuni esempi di applicazione del modello delle "5 Stelle"

181 5. TRASFormaZione Digitale E Riprogettazione ORGANIZZATIVA

$181 \quad 5.1 \quad$ Tecnologie digitali

$187 \quad 5.2$ Trasformazione digitale

$1895.3 \quad$ I potenziali benefici apportati dalla digital transformation 
$192 \quad 5.4 \quad$ Strategia digitale

$195 \quad 5.5$ Strategie digitali e cambiamento organizzativo

$200 \quad 5.5 .1 \quad$ Struttura

$205 \quad 5.5 .2$ Persone

$212 \quad 5.5 .3$ Processi

$219 \quad 5.5 .4$ Ricompense

$220 \quad 5.5 .5$ Esempi di applicazione del modello delle 5 Stelle al rapporto tra strategie digitali e sistema organizzativo

225 Postfazione

Luigi Maria Sicca

231 Riferimenti bibliografici

237 Indice delle figure

239 Indice delle tabelle

241 Hanno scritto nella Collana punto org 



\section{Riferimenti bibliografici}

AA.VV. (2014), Looking Forward. La trasformazione digitale, Supplemento al n. 12/2014 di Harvard Business Review Italia.

Acerbi, F., Assiani, S., De Carolis, A. (2019), Industria 4.0 e lavoro: le competenze necessarie in azienda e il metodo per valutarle, EconomyUp, 18 novembre.

Anthony, R.N., Hawkins, D.F., Macrì, D.M., Merchant, K.A. (2008), Sistemi di controllo - Analisi economiche per le decisioni aziendali, $3^{\circ}$ ed., Milano, McGraw-Hill.

Antonucci, Y.L., Fortune, A., Kirchmer, M. (2021), An examination of associations between business process management capabilities and the benefits of digitalization: all capabilities are not equal, Business Process Management Journal, 27(1), 124-144.

Argyris, C., Schön, D. (1995), Organizational Learning: Theory, method and practice, New York, Addison-Wesley.

Assolombarda (2015), Alla ricerca delle competenze 4.0, Analisi condotta in collaborazione da Assolombarda - Confindustria Milano Monza Brianza e l'Università di Milano Bicocca-Crisp, Milano, Assolombarda.

Barenfanger, R., Otto, B. (2015), Proposing a Capability Perspective on Digital Business Models, CBI '15: Proceedings of the 2015 IEEE 17th Conference on Business Informatics, Vol. 1, 17-25.

Barnard, C.I. (1948), Organization and Management, Cambridge, MA, Harvard University Press.

Barney, J.B. (1991), Firm Resources and Sustained Competitive Advantage, Journal of Management, 17(1), 99-120.

Bartezzaghi, E. (2010), L'organizzazione dell'impresa. Processi, Progetti, Conoscenza, Persone Milano, Etas.

Bartezzaghi, E., Della Rocca, G., (2019), “Tecnologia digitale, organizzazione e lavoro", in C. Dell' Aringa, P. Guerrieri (a cura di), Inclusione, Produttività, Crescita. Un'agenda per l'Italia a, Bologna, Il Mulino.

Bartezzaghi, E., Cagliano, R., Guerci, M., Gilardi, S., Canterino, F. (2020), Progettazione organizzativa 4.0: verso una rivisitazione dei principi sociotecnici, Studi organizzativi, vol. XXII, special issue, pp. 179-206. 
Betancourt, P., Mooney, J., Ross, J.W. (2015), Digital Innovation at Toyota Motor North America: Revamping the Role of IT, CISR Working Paper No. 403, Boston, MIT, Sloan School of Management.

Bonazzi, G. (2008), Storia del pensiero organizzativo, Milano, FrancoAngeli.

Bose, R. (2002), Customer relationship management: key components for IT success, Industrial Management E Data Systems, 102(2), 89-97.

Braga, A. (2017), Digital Transformation, Milano, Egea.

Burns, T., Stalker, G.M. (1961), The Management of Innovation, London, Tavistock.

Butler, R. (1996), Designing organizations: a decision making perspective, London, International Thomson Business Press.

Buttle, F., Maklan, S. (2019), Customer Relationship Management. Concepts and Technologies, London, Routledge.

Cagliano, R. (2010), "Processi aziendali, strutture organizzative e sistemi di gestione", in E. Bartezzaghi (a cura di), L'organizzazione dell'impresa. Processi, Progetti, Conoscenza, Persone, Milano, Rizzoli ETAS.

Capaldo, G. (2021), Il Business Process Management. Gestire i processi aziendali con un approccio olistico per creare valore e facilitare la trasformazione digitale, Milano, FrancoAngeli.

Capaldo, G., Volpe, A. (2021), Project Management. Principi e metodi, Milano, McGraw-Hill.

Chandler, A.D. (1962), Strategy and Structure: Chapters in the History of the American Industrial Enterprise, Cambridge, MA, MIT Press.

Chandler, A.D. (1977), The Visible Hand, Cambridge, MA, Belknap Press.

Child, J. (1973), Predicting and Understanding Organization Structure, Administrative Science Quarterly, 18(2), 168-185.

Corbucci, D. (2015), Agile project management, Milano, FrancoAngeli.

Costa, G., Gianecchini, M. (2019), Risorse umane, Milano, McGraw-Hill.

Costa, G., Gubitta, P. (2018), Organizzazione aziendale: mercati, gerarchie, convenzioni, Milano, McGraw-Hill.

Costa, G., Nacamulli, R.C. (1996), Manuale di organizzazione aziendale. Volume 1: Le teorie organizzative, Torino, UTET.

Cuneo, M. (2019), Essere leader per raggiungere il successo, www.mirkocuneo.it, 30 dicembre.

Daft, R.L. (2013), Organization theory and design, $12^{\text {th }}$ ed., Boston, MA, Cengage Learning.

Davenport, T.H., Spanyi, A. (2019), What Process Mining Is, and Why Companies Should Do It, Harvard Business Review, April 23.

de Bruin, T., Rosemann, M. (2007), Using the Delphi Technique to Identify BPM Capability Areas, ACIS 2007 Proceedings, 42, 643-653.

Dioguardi, G. (1986), Organizzazione come strategia, Torino, Isedi.

Elia, G., Margherita, A., Secundo, G. (2020), Impresa Digitale. Scenari, tecnologie e percorsi di trasformazione digitale, Milano, Egea. 
Fantoni, G., Al-Zubaidi, S.Q., Coli, E., Mazzei, D. (2021), Automating the process of method-time-measurement, International Journal of Productivity and Performance Management, 70(4), 958-982.

Feldman, M.S., Pentland B.T. (2003), Reconceptualizing organizational routines as a source of flexibility and change, Administrative Science Quarterly, 48(1), 94-118.

Fontana, F. (2010), Il sistema organizzativo aziendale, Milano, FrancoAngeli.

Ford, H., Crowther, S. (1922), My Life and Work, Garden City, Garden City Publishing Co.

Frigelli, U., a cura di, (2001), Il governo dei processi. Istruzioni per l'uso, Milano, Guerini e Associati.

Galbraith, J.R. (1971), Matrix Organization Designs, Business Horizon, February, p. 37.

Galbraith, J.R. (1977), Organization design, Reading, MA, Addison-Wesley.

Ganz, W. (2014), Welche Rolle spielen die Dienstleistungen in der Industrie 4.0?, relazione presentata al convegno "FES-Fachgespraøchs Industrie $4.0^{\prime \prime}$, Berlino, 8 ottobre.

Giddens, A. (1984), The Constitution of Society. Outline of the Theory of Structuration, Berkeley, Los Angeles, University of California Press.

Grandori, A. (1995), Organizzazione e comportamento economico, Bologna, Il Mulino.

Greiner, L.E. (1998), Evolution and Revolution as Organizations Grow, Harvard Business Review, May-June.

Gulati, R. Wohlgezogen, F., Zhelyazkov, P. (2012), The Two Facets of Collaboration: Cooperation and Coordination in Strategic Alliances, Academy of Management Annals, 6, 531-583.

Hammer, M., Champy, J. (1993), Reengineering the Corporation: A Manifesto for Business Revolution, Harper Business

Hannan, M.T., Freeman, J.H. (1977), The population ecology of organizations, American Journal of Sociology, 82(5), 929-964.

Highsmith, J. (2002), Agile Software Development Ecosystems, Boston, MA, Pearson Education Inc.

Ingrassia, R., (2001), Comunicazione organizzativa per l'amministrazione pubblica: il ruolo della comunicazione interna, Studi Organizzativi, 2-3, 35-64.

Isotta, F. (1989), "Le forme organizzative: evoluzione e progettazione", in M. Rispoli (a cura di), L'impresa industriale. Economia, tecnologia, management, Bologna, Il Mulino.

Jacobides, M.G., Sundararajan, A., Van Alstyne, M. (2019), Platforms and Ecosystems: enabling the digital economy, Ginevra, World Economic Forum. 
Jones, G. (2010), Organizational Theory, Design and Change, New York, Pearson education, Prentice Hall.

Joss, J.W., Beath, C.M., Mocker, M. (2019), Designed for Digital: How to Architect Your Business for Sustained Success, Cambridge, MA, MIT Press.

Kane, G.C., Phillips, A.N., Copulsky, J.R., Andrus G.R. (2019), The Technology Fallacy. How People Are the Real Key to Digital Transformation, Cambridge, MA, MIT Press.

Kerpedzhiev, G.D., Lehnert, M. Röglinger, M. (2016), The Future of Business Process Management in the Future of Work, ECIS 2016 Proceedings, Research Papers, 81, 1-18.

Kerpedzhiev, G.D., König, U.M., Röglinger, M., Rosemann M. (2020), An Exploration into Future Business Process Management Capabilities in View of Digitalization. Results from a Delphi Study, Business \& Information Systems Engineering, 63(2), 83-96.

Kurtz, C. (2014), Mensch, Maschine und die Zukunft der Industriearbeit, relazione presentata al convegno "Maschinen entscheiden: vom Cognitive Computing zu autonomen Systemen", Monaco, 21 novembre.

Lawrence, P., Lorsch, J. (1967), Differentiation and Integration in Complex Organizations, Administrative Science Quarterly, 12, 1-30.

March, J.G., Simon, H. (1958), Organizations, New York, John Wiley and Sons.

Mayo, E. (1933), The human problems of an industrial civilization, Cambridge, MA, Harvard University Press.

McFarland, W. (2015), Managers in the Digital Age Need to Stay Human, Harvard Business Review, 17 giugno.

Migliarese, P. (2010), Lezioni di sistemi organizzativi aziendali, Roma, Aracne.

Morchio, M. (2014), "Il nuovo paradigma della strategia”, in AA.VV., Looking Forward. La Trasformazione Digitale. Supplemento al n. 12/2014 di Harvard Business Review Italia.

Nonaka, I., Takeuchi, H. (1995), The Knowledge Creating Company, Oxford, Oxford University Press.

Orlikowski, W.J. (2000), Using Technology and Constituting Structures: A Practice Lens for Studying Technology in Organizations, Organization Science, 11(4), 404-428.

Ouchi, W.G. (1977), The Relationship Between Organizational Structure and Organizational Control, Administrative Science Quarterly, 22(1), 95-113.

Pagliai, A., Sperimborgo, S. (2014), "Accelerare la trasformazione", in AA.VV., Looking Forward. La Trasformazione Digitale. Aggiungere tecnologia al business per ottenere l'Effetto Moltiplicatore. Supplemento al n. 12/2014 di Harvard Business Review Italia.

Pascale, R.T., Athos, A.G. (1981), The art of Japanese management, New York, Simon \& Schuster. 
Paunov, C., Planes-Satorra S. (2019), How are digital technologies changing innovation? Evidence from agriculture, the automotive industry and retail, OECD Science, Technology And Industry Policy Papers, 74, Paris. OECD Publishing.

Perrow, C. (1967), A Framework for the Comparative Analysis of Organizations, American Sociological Review, 32(2), 194-208.

Pfeffer, J., Salancik, G. (1978), The External Control of Organizations: A Resource Dependence Perspective, New York, Harper \& Row.

Polanyi, M., (1958), Personal Knowledge. Towards Post-Critical Philosophy, London, Routledge \& Kegan Paul.

Powell, W.M. (1990), "Neither Market nor Hierarchy; Network Forms of Organization", in B.M. Staw, L.L. Cummings (Eds.), Research in Organizational Behavior, Greenwich, CT, JAI Press, 295-336.

Rebora, G. (2001), Manuale di organizzazione aziendale, Roma, Carocci.

Rebora, G. (2017), Scienza dell'organizzazione: il design di strutture, processi e ruoli, Roma, Carocci.

Reinartz, W., Krafft, M., Hoyer, W. (2004), The Customer Relationship Management Process: Its Measurement and Impact on Performance, Journal of Marketing Research, 41(3), 293-305.

Reinartz, W.J., Kumar, V. (2000), On the Profitability of Long-Life Customers in a Noncontractual Setting: An Empirical Investigation and Implications for Marketing, Journal of Marketing, 64(4), 17-35.

Ronchi, M., Ciancia, M. (2019), Digital transformation, Milano, FrancoAngeli. Rummler, G.A., Brache, A.P. (2013), Improving Performance. How to Manage the White Space in the Organization Chart, 3rd edition, San Francisco, Jossey-Bass.

Salas, E., Dickinson, T.L., Converse, S.A., Tannenbaum, S.I. (1992), "Toward an understanding of team performance and training", in R. Swezey, E. Salas (Eds.), Teams: Their Training and Performance, Norwood, NJ, Ablex Publishing Corporation, pp. 3-29.

Scott, W.R. (1981), Organizations: rational, natural and open systems, Englewood Cliffs, Prentice-Hall.

Seghezzi F. (2015), Come cambia il lavoro nell'Industry 4.0?, Woking Paper ADAPT, n. 172.

Seghezzi F. (2016), Lavoro e relazioni industriali in Industry 4.0, Diritto delle Relazioni Industriali, n. 1.

Seiler, J.A. (1967), Systems analysis in organizational behavior, Homewood, IL, Irwin.

Senge, P.M. (1990), “The art and practice of the learning organization”, in M. Ray, A. Rinzler (eds.), The new paradigm in business: Emerging strategies for leadership and organizational change, Los Angeles, Tarcher, 126-138.

Senge, P.M. (2010), The Fifth Discipline: The Art E Practice of The Learning Organization, New York, Currency. 
Simon, H.A. (1947), Administrative behaviour, New York, McMillian.

Smith, A. (1976), La ricchezza delle nazioni, Roma, Newton Compton Editori.

Spender, J.-C., Grant, R.M. (1996), Knowledge and the firm: Overview, Strategic Management Journal, 17, 5-9.

Stjepić, A-M., Ivančić, L., Vugec, D.S. (2019), Mastering digital transformation through business process management: Investigating alignments, goals, orchestration, and roles, Journal of Entrepreneurship Management and Innovation, 16(1), 41-74.

Thompson, J.D. (1967), Organizations in Action: Social Science Bases of Administrative Theory, New Brunswick, NJ, Transaction Publishers.

Trist, E., Bamforth, W. (1951), Some Social and Psychological Consequences of the Long Wall Method of Coal-Getting, Human Relations, 4, 3-38.

Trombetta, S., Chiadò, C., Collina L., Giordano, L. (2014), “Verso un'organizzazione digitally skilled", in AA.VV., Looking Forward. La Trasformazione Digitale. Supplemento al n. 12/2014 di Harvard Business Review Italia.

Venier, F., (2017), Trasformazione digitale e capacità organizzativa. Le aziende italiane e la sfida del cambiamento, Trieste, EUT Edizioni Università di Trieste.

Volberda, H., Lewin, A. (2003), Guest Editors' Introduction Co-evolutionary Dynamics Within and Between Firms: From Evolution to Co-evolution, Journal of Management Studies, 40, 2111-2136.

Westerman, G., Bonnet, D., McAfee, A., (2014), Leading Digital: Turning Technology into Business Transformation, Boston, Harvard Business Press.

Williamson, O.E. (1975), Markets and hierarchies, analysis and antitrust implications: A study in the economics of internal organization, New York, Free Press.

Williamson, O.E. (1985), The economic institutions of capitalism: firms, markets, relational contracting, New York, Free Press.

Williamson, O.E. (1986), Economic organization: firms, markets, and policy control, New York, New York University Press, 1986.

Womack, J.P., Jones, D.T., Ross, D. (1991), The machine that changed the world, New York, Harper Perennial.

Woodward, J. (1965), Industrial organization, London, Oxford University Press.

World Economic Forum (2018), HR4.0: Shaping People Strategies in the Fourth Industrial Revolution, Geneva, WEF. 
Indice delle figure

Fig. 2.1 Le dimensioni della tecnologia 62

Fig. 2.2 Lo star model 64

Fig. 2.3 Il continuum delle strutture organizzative $\quad 72$

Fig. 2.4 Il ciclo di conversione della conoscenza 84

Fig. 3.1 Il sistema e le variabili organizzative 96

Fig. 3.2 L'interdipendenza tra i livelli del sistema organizzativo 105

Fig. 3.3 Il modello delle "5 stelle" 108

Fig. 4.1 Esempio di struttura organizzativa funzionale 123

Fig. 4.2 Esempio di struttura divisionale per prodotti 125

Fig. 4.3 Esempio di struttura divisionale per aree geografiche

Fig. 4.4 Esempio di struttura divisionale per segmenti di clientela

Fig. 4.5 Esempio di struttura divisionalizzata $\quad 127$

Fig. 4.6 Esempio di struttura funzionale 128

Fig. 4.7 La struttura ibrida adatta al Caso $1 \quad 130$

Fig. 4.8 La struttura ibrida adatta al Caso 2

Fig. 4.9 La struttura più adatta al Caso $3 \quad 134$

Fig. 4.10 Organizzazione della commessa 135

Fig. 4.11 Esempio di struttura per progetti 137

Fig. 4.12 Esempio di struttura a matrice 138

Fig. 4.13 Esempi di strutture orientate ai processi 141

Fig. 4.14 Relazione tra obiettivi "dominanti" e struttura organizzativa

Fig. 4.15 Fasi del ciclo di vita e strutture organizzative 
Fig. 4.16 Esempio di Work Breakdown Structure 167

Fig. 4.17 Esempio di Flow chart o diagramma di flusso 168

Fig. 4.18 La matrice delle responsabilità 169

Fig. 4.19 Il contesto e la complessità relazionale del ruolo 
Indice delle tabelle

Tab.2.1 Complessità tecnologica e struttura organizzativa 61

Tab. 2.2 I principali contributi sulla progettazione organizzativa

Tab. 4.1 Tipologia di relazione e scala di misurazione del legame $\quad 157$

Tab. 4.2 Tipologia di area di responsabilità e legame $\quad 159$

Tab. 4.3 Esempio di calcolo del fabbisogno di personale 162

Tab. 4.4 Fasi e strumenti per la descrizione dei processi 165

Tab. 4.5 Possibili criteri di accorpamento dei compiti $\quad 175$

Tab. 4.6 Alcuni esempi di applicazione del modello delle 5 Stelle

Tab. 5.1 I vantaggi delle tecnologie digitali per alcuni processi aziendali

Tab. 5.2 Alcuni esempi di applicazione del modello delle 5 Stelle alle strategie digitali 



\section{Hanno scritto nella Collana punto org}

www.puntoorg.net

1. L.M. Sicca (a cura di)', Leggere e scrivere organizzazioni. Estetica, umanesimo e conoscenze manageriali (con postfazione di F. Piro), 2010.

2. L.M. Sicca, Alla fonte dei saperi manageriali. Il ruolo della musica nella ricerca per l'innovazione e per la formazione delle risorse umane, 2012.

3. A. Di Scipio, Pensare le tecnologie del suono e della musica (con prefazione di R. Diana), 2012.

4. R. Musto, Scienza Natura Cambiamento (con prefazione di M. Nicode$\mathrm{mi}), 2012$.

5. R. Musto, Novalis. L'assoluto e le cose (con prefazione di C. Albarella e postfazione di G. Imbruglia), 2013.

6. Aa.Vv.", I linguaggi dell'organizzare. Musica e testo tra dono e disinteresse, 2013.

7. B. Masiello, Fiducia nelle reti. Strategie per la crescita nei mercati internazionali delle PMI (con prefazione di F. Izzo), 2013.

8. Aa.Vv. ${ }^{\text {II }}$, Tavola rotonda. Umanesimo del management attraverso gli occhi dell'altro, 2013.

9. M. Calcagno, Narrare terre di mezzo. Management arte design (con prefazione di S. Faccipieri e postfazione di A. Comacchio), 2013.

10. R. Diana, Disappartenenza dell'To. Filosofia e musica verso Samuel Beckett (con prefazione di L.M. Sicca), 2014.

${ }^{1}$ Con scritti di Per Olof Berg e Kristian Kreiner, Robert W. Witkin, Barbara Czarniawska e Carl Rhodes, Ken Starkey e Sue Tempest, John Hendry, Karin Knorr Cetina.

" Con scritti di Luigi Maria Sicca, Umberto di Porzio, Rosario Diana, Agostino Di Scipio, Mariella De Simone, Bernardo Maria Sannino, Chiara Mallozzi, Lorenzo Pone, Giancarlo Turaccio.

"I Con scritti di Luigi Maria Sicca, Francesco Izzo, Maura Striano, Giulia Dell'Aquila, Felice Casucci, Francesco Perillo, Rosario Diana, Paola Giampaolo, Davide Bizjak, Gilberto-Antonio Marselli, Franco Vitelli, Maria Rosaria Napolitano. 
11. Aa.Vv. ${ }^{\text {IV }}$, Sergio Piro. Maestri e allievi, 2014.

12. F.D. Perillo (a cura di) ${ }^{\mathrm{v}}$, Impresa imperfetta, 2014.

13. L.M. Sicca, L. Zan (a cura di) ${ }^{\mathrm{v}}$, Management Arti Culture. Resoconto del primo anno del GSA - Accademia Italiana di Economia Aziendale, 2014.

14. M. Iaccarino, Un mondo assetato. Come il bisogno di acqua plasma la civiltà (con prefazione di F.P. Casavola e postfazione di A. Giannola), 2015.

15. F. Piro, Manuale di educazione al pensiero critico. Comprendere e argomentare (con prefazione di T. De Mauro), 2015.

16. F. D'Errico, Fuor di metafora. Sette osservazioni sull'improvvisazione musicale (con prefazione di P. de Vita e postfazione di M. Maldonato), 2015.

17. E. Mollona, Computer Simulation in Social Sciences. A Logic of Enquiry (with a preface by L.M. Sicca, a foreword by G. Colombo and an afterword by D. Secchi), 2015.

18.S. Oliverio, L.M. Sicca, P. Valerio ${ }^{\mathrm{vII}}$, Transformare le pratiche nelle organizzazioni di lavoro e di pensiero (con prefazione di G. Manfredi), 2015.

19. P. Valerio, C. Bertolazzi, P. Marcasciano (a cura di) ${ }^{\mathrm{v}}$, Transformare l'organizzazione dei luoghi di detenzione. Persone transgender e gender nonconforming tra diritti e identità (con prefazione di L.M. Sicca), 2016.

iv Con scritti di Giuseppe Cantillo, Tullio De Mauro, Aldo Masullo, Mariapaola Fimiani, Teresa Capacchione, Antonio Mancini, Roberto Beneduce, Enrico De Notaris, Fulvio Marone, Dario Stefano Dell'aquila, Luigi Maria Sicca, Francesco Piro.

v Con scritti di Pier Luigi Celli, Eugenio Mazzarella, Enzo Rullani, Luigi Maria Sicca, Francesco Varanini.

vi Con scritti di Stefano Baia Curioni, Paola Dubinie Ludovica Leone, Sara Bonini Baraldi e Luca Zan, Monica Calcagno e Luigi M. Sicca, Donata Collodi, Francesco Crisci e Andrea Moretti, Roberto Ferrari e Alessandro Hinna, Francesco Giaccari, Francesca Imperiale e Valentina Terlizzi, Daniele Goldoni, Pamela Palmi.

vi Con scritti di Anna Lisa Amodeo, Christian Ballarin, Davide Bizjak, Ilaria Boncori e Paolo Fazzari, Rossella Bonito Oliva, Simone Cangelosi, Marco De Giorgi, Guglielmo Faldetta, Vittoria Fiorelli, Stefano Maltese, Porpora Marcasciano, Piergiorgio Masi, Antonia Monopoli e Chiara Repetto, Andrea Morniroli, Edoardo Mollona, Cristiano Scandurra, Luca Solari, Maria Spanò, Maria Gigliola Toniollo.

viII Con scritti di: Paolo Valerio, Giuseppe Ferraro, Carmen Bertolazzi, Alexander Hochdorn, Porpora Marcasciano, Luca Chianura, Damiana Massara, Daniela A. Nadalin, Adriana Godano, Luca Chianura, Vittoria Colonna, Elia De Caro, Tito Flagella, Anna Lorenzetti. 
20. M.R. Napolitano, V. Marino (a cura di) ${ }^{\mathrm{Ix}}$, Cultural Heritage e Made In Italy. Casi ed esperienze di marketing internazionale (con prefazione di G. Volpe e postfazione di A. Mattiacci), 2016.

21. M. Lusiani, Discourses of Planning (with a preface by L. Zan and an afterword by A. Langley), 2016.

22. F.D. Perillo, Simposio manageriale (con prefazione di A. Masullo e postfazione di P.L. Celli), 2016.

23. P. Ferri, I commissariamenti nel settore culturale italiano. Obiettivi, azioni, risultati (con prefazione di L. Zan e postfazione di G. Grossi), 2016.

24. L. Pareschi, Controcampo letterario. Strategie di intermediazione e accesso all'industria editoriale (con prefazione di P. Dubini e postfazione di G. Colombo), 2016.

25. G.-A. Marselli, Mondo contadino e azione meridionalista. L'esperienza del Gruppo Rossi-Doria a Portici (con prefazione di E. Mazzetti e postfazione di F. Vitelli), 2016.

26. F. Accardix, Risk and Control Governance. A value-creation perspective (with a preface by A. De Nicola; an introduction by V. Atella and an afterword by S. Bozzolan), 2017.

27. I. Boncori (ed) ${ }^{\mathrm{x}}, L G B T+$ Perspectives. The University of Essex Reader (with a foreword by A. Forster), 2017.

28. A. Papa, “... Una cappella cavata dentro il monte...". Storia minima del complesso monastico di S. Lucia al Monte (con prefazione di L. d'Alessandro), 2017.

זx Con scritti di Loretta Battaglia, Giuseppe Bertoli, Roberta Biandolino, Michelle Bonera, Enrico Bonetti, Mauro Cavallone, Elena Cedrola, Marta Cerquetti, Maria Chiarvesio, Anna Codini, Emanuela Conti, Eleonora Di Maria, Barbara Francioni, Antonella Garofano, Francesco Izzo, Giulia Lanzilli, Gaetano Macario, Giulio Maggiore, Francesca Magno, Vittoria Marino, Barbara Masiello, Michela Matarazzo, Alberto Mattiacci, Marta Maria Montella, Fabio Musso, Maria Rosaria Napolitano, Alessandro Pagano, Tonino Pencarelli, Giovanna Pegan, Michele Quintano, Riccardo Resciniti, Marcello Risitano, Angelo Riviezzo, Savino Santovito, Elisabetta Savelli, Michele Simoni, Annarita Sorrentino, Raffaella Tabacco, Donata Vianelli.

${ }^{x}$ Con scritti di Roberto Rosato, Nicoletta Mincato, Carlo Nicoletti, Paolo De Paolis, Alessandro Salibra Bove.

хг Con scritti di Alison J. Taylor-Lamb, Jamie Raines, Thomas Currid and Carl Chandra, Martin Harrison and Peter Martin, Rainer Shulze, Fleur Jeans and Teresa Eade, Tuesday Wats, Amy Anderson, Sco Lawley. 
29. R. Diana, L.M. Sicca, G. Turaccio ${ }^{\mathrm{xII}}$, Risonanze. Organizzazione, musica, scienze (con prefazione di A. Strati e postfazione di A. Solbiati), 2017.

30. F. D'Errico, Armonia funzionale e modalità. Rudimenti per l'improvvisazione a indirizzo jazzistico (con introduzione di F. Piro e prefazione di R. Grisley), 2017.

31. M. Calcagno, Interpreting Innovation. Design Creativity Art (with a foreword by F. Izzo; preface by A. Moretti and an afterword by J. Metelmann), 2017.

32. G. Balirano, Gardai \& Badfellas: The Discursive Construction of Organised Crime in the Irish Media (with a foreword by L.M. Sicca), 2017.

33. M.C. Mason, A. Moretti, Tattoo Management. Mercati, attori, valore, 2017.

34. P. Testa, Innovazione del modello di business. Le dimensioni latenti nella letteratura di management (con prefazione di L. Cantone), 2017.

35. L. Massa, Viva 'o re! Municipio e dintorni (con introduzione di L.M. Sicca; prefazione di E. Borgonovi e postfazione di C. Mochi Sismondi), 2017.

36. F. Pavan, Memini. Piccole storie di storia della musica (con introduzione di E. Mazzarella; prefazione di R. Alessandrini e postfazione di V. Moroni), 2017.

37. C. Mallozzi, D. Tortora ${ }^{\mathrm{xII}}$, La bottega del suono. Mario Bertoncini. Maestri e allievi (con prefazione di M. Nicodemi e postfazione di L.M. Sicca), 2017.

38. G. Melis, Collaborazione e apprendimento nei processi di co-creazione di valore. Il caso delle destinazioni turistiche (con prefazione di M.R. Napolitano e postfazione di B. Argiolas), 2018.

xII Con scritti di Davide Bizjak, Dario Casillo, Rosario Diana, Umberto Di Porzio, Agostino Di Scipio, Chiara Mallozzi, Mario Nicodemi, Lorenzo Pone, Rosalba Quindici, Sonia Ritondale, Tommaso Rossi, Bernardo Maria Sannino, Luigi Maria Sicca, Cristian Sommaiuolo, Giancarlo Turaccio, Paolo Valerio.

xIII Con scritti di Mario Bertoncini, Davide Bizjak, Gianmario Borio, Pietro Cavallotti, Andrew Culver, Francesco D'Errico, Charles de Mestral, Michelangelo Lupone, Chiara Mallozzi, Alessandro Mastropietro, Mario Nicodemi, Luigino Pizzaleo, Lorenzo Pone, Ingrid Pustijanac, John Rea, Bernardo Maria Sannino, Luigi Maria Sicca, Daniela Tortora. 
39. G. Viglia, A.C. Invernizzi, Il ruolo dell'hubris nella gestione imprenditoriale (con prefazione di C. Mauri), 2018.

40. T. Russo Spena, C. Mele, Practising innovation. A Sociomaterial View (with a foreword by E. Gummesson; preface by J. Spohrer and an afterword by P. Stampacchia), 2018.

41. I. Boncori, Race, Ethnicity and Inclusion. The University of Essex Reader (with a foreword by A. Forster and a postface by M. Śliwa), 2018.

42. K.E. Russo, The Evaluation of Risk in Institutional and Newspaper Discourse. The Case of Climate Change and Migration (with a preface by G. Bettini), 2018.

43. R. Pera, When Consumers get Creative. Cocreation in the Individual and Collective Realm (with a preface by D. Dalli), 2018.

44. F. Piro, L.M. Sicca, P. Maturi, M. Squillante, M. Striano (a cura di) ${ }^{\mathrm{xiv}}$, Sfide didattiche. Il pensiero critico nella scuola e nell'università (con prefazione di F. Sabatini), 2018.

45. R. Quaglia, Bravi ma basta! Su certe premesse, promesse e catastrofi culturali (con introduzione di L.M. Sicca; prefazione di J. Mills e postfazione di F. Barca), 2018.

46. B. Czarniawska, La narrazione nelle scienze sociali, I edizione italiana a cura di L.M. Sicca, F. Piro, I. Boncori, 2018.

47. F. Longobardi, Le affinità del lessico, 2018.

48. G. Calogero, L'abbiccì della democrazia. E altri scritti, (a cura di) R. Trombelli (con una testimonianza di G. Sasso), 2018.

49. V. Fiorelli (a cura di $)^{\mathrm{xv}}$, Margini e confini. Attraversamenti di metodi e linguaggi tra comunicazione, didattica e possibilità della ricerca (con prefazione di L. d'Alessandro), 2018.

50. G. Cundari, Il mondo: una bella prigione? Riflessioni geografiche, 2018.

xiv Con scritti di Maura Striano, Rosaria Capobianco e Maria Rita Petitti, Francesco Piro, Roberta Gimigliano, Monica Mollo, Gerarda Fattoruso, Maria Incoronata Fredella, Maria Grazia Olivieri, Massimo Squillante e Antonia Travaglione, Pietro Maturi, Fabio Maria Risolo, Luca Marano, Luigi Maria Sicca, Giuseppe Recinto, Mario Nicodemi, Chiara Mallozzi e Luigi Marolda, Luigi Proserpio, Davide Bizjak, Paolo Canonico, Stefano Consiglio, Ernesto De Nito e Teresa Anna Rita Gentile, Natascia Villani.

xv Con scritti di Giuliano Amato, Gianluca Bocchi, Massimo Abdallah Cozzolino, Diego Davide, Lucia Donsi, Amedeo Feniello, Vittoria Fiorelli, Luigi Manconi, Roberta Morosini, Gianmarco Pisa, Ciro Pizzo, Leopoldo Repola, Stefano Rodotà, Francesco Varanini. 
51. M. Nicotra, Il canto dei sireni. Invenzioni trans/singolari e psicoanalisi lacaniana (con prefazione di A. Vicens e postfazione di P. Guazzo), 2019.

52. L. Marano, Come parlano i giovani. Una indagine di sociolinguistica urbana (con prefazione di P. Maturi), 2019.

53. M. Floris, R. Tronci, C. Dessì, A. Dettori, Imprese familiari e imprenditorialità. La sfida del cambiamento (con prefazione di F. Cabiddu), 2019.

54. T.T. Lennerfors, L. Mitchell (eds) ${ }^{\mathrm{xv}}$, SCOS. Searching Collectively for Our Soul, 2019.

55. Aa.Vv. ${ }^{\text {xvII }}$, Lucio Sicca. Maestri e allievi, 2019.

56. E. Sacerdote ${ }^{\text {хvш }}$, Breviario sul pensiero strategico. Discorsi e percorsi per conquistare il futuro migliore, 2019.

57. M.E. Santagati, L. Zan (a cura di) ${ }^{\mathrm{xx}}$, Imprenditorialità e settore museale. Esperienze e prospettive di cambiamento, 2019.

58. F.D. Perillo, D. Bizjak, L'impresa nello zaino. La Elmeco da vico San Liborio al mondo, 2019.

59. F.D. Perillo, D. Bizjak, R.A. Tundo, The Company in the Backpack. Elmeco's, from Vico San Liborio to the World, 2019.

${ }^{x v i}$ Con scritti di Beatriz Acevedo, Omar Aktouf, Noortje van Amsterdam, Per Olof Berg, Davide Bizjak, Ilaria Boncori, Jo Brewis, Peter Case, Patricia Ehrensal, Ken Ehrensal, Hugo Gaggiotti, Silvia Gherardi, Claes Gustafsson, Campbell Jones, Nina Kivinen, Monika Kostera, Kristian J Kreiner, Thomas Taro Lennerfors, Marcus Lindahl, Steve Linstead, Tomek Ludwicki, Takashi Majima, Laura Mitchell, Albert J. Mills, Jean Helms Mills, Luc Peters, Alf Rehn, Harriet Shortt, Luigi Maria Sicca, Antonio Strati, Sam Warren, Robert Witkin, Anthony R. Yue, Peter Zackariasson.

${ }^{x v I I}$ Con scritti di Luigi Guatri, Giovanni Zanetti, Adriano Giannola, Franco Amatori, Renato Mele, Francesco Testa e Riccardo Mercurio; Enzo Rullani, Paolo de Vita, Salvatore Vicari, Luigi Cantone, Pierpaolo Testa e Vincenzo Basile; Marco Ferretti, Maria Rosaria Napolitano, Francesco Izzo, Enrico Bonetti, Antonio Capaldo, Paolo Calvosa, Davide Bizjak.

xvır Con scritti di Stefano De Luca e Guido Solza.

xix Con scritti di Chiara Bombardieri, Paola Degli Esposti, Mariagabriella Fornasiero, Valentina Galloni, Alessandro Gazzotti, Claudia Giordani, Federica Guidi, Annalisa Managlia, Umberto Mossetti, Michele Recanatini, Elisa Schiavina, Stefania Spaggiari, Patrizia Tomba, Cinzia Vecchi. 
60. M. Castro Espín ${ }^{\mathrm{xx}}$, Persone transessuali a Cuba (con prefazione di L.M. Sicca), 2019.

61. G. van Wulfen, La spedizione per innovare. Un kit di strumenti visual per far partire l'innovazione (I edizione italiana a cura di M.V. Colucci, A. Forciniti, L. Migliola, L. Rossi), 2019.

62. P. Senge ${ }^{\mathrm{xx}}$, La quinta disciplina: l'arte e la pratica dell'apprendimento organizzativo (ed 2006), (edizione italiana a cura di L.M. Sicca), 2019.

63. V. Basile, Marketing Performance Measurement in Fmcg Share of Wallet in Retailing Industry (with a preface by L. Cantone), 2020.

64. A. Moretti, R. Tabacco, I Menuzzo e C AME. Una storia di passione per il prodotto, i clienti e le relazioni (con postfazione di R. Samiolo), 2020.

65. B. Masiello, Il lato oscuro delle reti. Strategie d'impresa e capitale sociale (con introduzione di F. Izzo), 2020.

66. F. Izzo, B. Masiello, Le fabbriche bianche. I processi di innovazione nelle imprese creative, 2020.

67. D. Salvatore, Saperi manageriali, crescita sostenibile e sud interno. Quali contributi possono offrire gli studi di organizzazione aziendale per una crescita economica sostenibile?, 2020.

68. P. Canonico, Understanding the role of knowledge in project settings, 2020.

69. I. Boncori, T. Loughran ${ }^{\mathrm{xxI}}$, Health and Wellbeing. The University of Essex Reader (with a preface by A. Forster), 2020.

xx Con scritti di Mario Bottone e Paolo Valerio; Christian Ballarin; Davide Bizjak e Cristiano Scandurra; Rossella Bonito Oliva; Giuseppe Burgio; Porpora Marcasciano; Mary Nicotra; Alessandra Riccio.

xxi Con scritti di Luigi Maria Sicca, Edoardo Mollona e Stefano Armenia; Nicola Andreottola, Simona Capecelatro, Davide Coppola; Sergio Barile e Francesca Iandolo; Mariavittoria Cicellin e Stefano Consiglio; Corrado Cuccurullo; Davide Bizjak, Paolo Canonico e Vito Lasala; Marcello Martinez e Mario Pezzillo Iacono; Stefano Mazzoleni e Francesco Giannino; Mario Nicodemi; Francesco Perillo; Francesco Piro; Apollonia Striano.

xxII Con scritti di Anthony Forster; Ilaria Boncori and Tracey Loughran; Martyna Śliwa; Charlie V.L. Smith; Lauren O'Connell; Abby Shovlin; Peter J. Martin and Edward M. Holt; Nileema Vaswani and Ilaria Boncori; Filippo Sinicato; Jaime Lindsey; Ewen Speed, Susan McPherson and Peter Beresford; Francine Bailey; Sean Seeger. 
70. J. Altmanova, L. Cannavacciuolo, M. Ottaiano, K. Russo (a cura di) ${ }^{\text {xxII }}$, Across the University. Linguaggi, narrazioni, rappresentazioni del mondo accademico, 2020.

71. M.T. Como, La Sala degli Angeli nel complesso conventuale di Suor Orsola Benincasa. Per una storia della costruzione (con prefazione di L. d'Alessandro), 2020.

72. S. Cardone, Breviario di Pedagogia Teatrale (con prefazione di A. Bergamo), 2020.

73. E. Sacerdote, Legendary brands. Valutazioni e riflessioni strategiche, 2020.

74. C. Bruno, Gestire l'industria dell'emozione. Un'analisi manageriale dell'attività dei teatri lirici (con prefazione di G. Fraquelli), 2020.

75. S. Consiglio, M. D'Isanto, La cultura che cambia (con premessa di E. D’Errico, prefazione di C. Borgomeo e postfazione di G. Volpe), 2020.

76. G. Nolfe, L.M. Sicca ${ }^{\text {xxiv }}$, Mobbing. Narrazioni individuali e organizzative (con prefazione di B. Czarniawska e postfazione di B. Poggio), 2020.

77. S. Armenia, The value of Systems Thinking and System Dynamics in the management of complex organizations. A selection of case studies (with a preface by E. Mollona), 2020.

78. B. Czarniawska, Per una teoria dell'organizzazione, edizione italiana a cura di L.M. Sicca, 2020.

79. P. Ventrella, Ludebat Bellerofontes. Vita $\mathcal{E}$ Opere di Bellerofonte Castaldi, liutista modenese (con prefazione di F. Pavan e postfazione di D. Cantalupi), 2020.

xxII Con scritti di Augusto Guarino, Maria Laudando, Maria Alessandra Giovannini, Giovanni Rotiroti, Ruth Amar, Valeria Sperti, Andrea Pezzè, Irma Carannante, Oriana Palusci, Antonio Saccone, Germana Volpe, Laura Cannavacciuolo, Giuseppina Notaro, Fabio Rodríguez Amaya, M. Cristina Lombardi, Marco Ottaiano, Franco Paris, Francesca De Cesare, Katherine Russo, Angela Buono, Anna Mongibello, Giovanni Agresti, Jana Altmanova, Giuliana Regnoli, Rosario Gallone, Vincenzo Bavaro, Federico Pio Gentile.

xxiv Con scritti di Giovanni Nolfe, Luigi Maria Sicca, Gemma Zontini, Christophe Dejours; Giuseppe Recinto, Raffaello Santagata de castro e Fabio Dell'Aversana, Mariapia Garavaglia; Luca Solari, Enzo Cordaro. 
80. L.M. Sicca, D. Borrelli, D. Napolitano (a cura di) ${ }^{x \times v}$, Senza valore (con prefazione di E. Mazzarella), 2021.

81. L. Limoccia, Disobbedienza civile alle leggi ingiuste ${ }^{\text {xxv }}$ (con prefazione di L.M. Sicca), 2021.

82. G. Faldetta, Il dono nelle organizzazioni aziendali (con prefazione di L. Bruni e postfazione di A. Cossetta e S. Labate), 2021.

83. N. Tartaglia, G. Antonelli, Innovare senza brevettare. Resistenze delle start up italiane, 2021.

84. G.R. Greco, Management della scienza e trasferimento di conoscenza. Principi modelli e limiti, 2021.

85. C. Canoro (a cura di) ${ }^{x x v i}$, Patrimonio culturale: passaporto per il futuro. Dive in the past, 2021.

86. U. Avanzi, M. Mocchi, E. Sacerdote, Il museo dialogante. Dall'ascolto alla co-creazione con il visit-attore (con introduzione di L.M. Sicca, prefazione di M. Coppola e postfazione di C. Sacerdoti), 2021.

87. G. D’alessio, La Biochimica di una vita. Un'autobiografia tra scienza ed emozioni, 2021.

88. F. Varanini, Perché posso dirmi formatore (con prefazione di L.M.Sicca), 2021.

xxv Con scritti di Alberto Abruzzese, Angelo Baccelloni, Davide Borrelli, Giovanni Costa, Gerarda Fattoruso, Andrea Fumagalli, Daniele Garritano, Anna Giannetti, Daniele Goldoni, Carlo Grassi, Domenico Napolitano, Mario Nicodemi, Maria Grazia Olivieri, Enzo Rullani, Luigi Maria Sicca, Massimo Squillante, Marialuisa Stazio, Marcello Traiola, Luca Zan.

xavi Con scritti di Don Tonino Palmese, Raffaele Nogaro, Carmen Capolupo, Enrico Euli, Mons. Francesco Savino, Giuseppe Cataldi, Gianni Cerchia, Giuseppe Limone, Rosaria de Marco, Francuccio Gesualdi, Gianmarco Pisa, Lombardi - Renato Salvetti, Gino Magurno, Enrico Peyretti, Giuseppe Cotturri, Guglielmo Faldetta, Luigi Maria Sicca, Deborah Gervasi, Davide Bizjak, Edoardo Mollona, Fedele Lizzi, Franco Pezzella, Massimo Bray, Giacomo Di Gennaro, Gianni Massa, Gianluca Medas, Carlo Crespellani Porcella, Silvano Tagliagambe, Valdo Spini, Nunzio Cennamo, Carla D'Antò, Carlo Patrizio, Patrizio Gonnella, Stefania Di Leo, Miguel Elias Sanchez Sanchez, Pedro En Rodríguez, Raffaele Gorgoni, Antonio Vitolo, Pietro Folena, Donatella Trotta, Paolo Naso, Nicola Lettieri, Fiammetta Fanizza, Cesare Moreno, Luca Signorini, Carmine Nardone, Silvia Tessitore, Stefania Caiazzo, Arturo De Vivo, Stefano Consiglio, Luisa Franzese, Fabio Giuliani, Gianfranco Nappi, Mauro Sciarelli.

xxvi Con scritti di Roberto Petriaggi, Filomena Lucci, Gabriele Gomez de Ayala, Enrico Gallocchio, Cristina Canoro, Fabio Bruno, Marco Cozza e Francesco Izzo. 
Finito di stampare nel mese di luglio 2021 dalla Vulcanica srl - Nola (NA) 